\title{
ON FUNCTIONS ANALYTIC IN A HALF-PLANE
}

BY

DANIEL WATERMAN

1. In the study of the behavior of functions analytic in the unit circle, the class $H_{p}$ of those functions $\phi(z)$ such that

$$
\int_{0}^{2 \pi}\left|\phi\left(\rho e^{i \theta}\right)\right|^{p} d \theta
$$

is uniformly bounded for $\rho<1$ is of particular importance. For $p>1, H_{p}$ is just the class of power series whose real parts are the Poisson integrals of functions in class $L_{p}$. The important role these classes play in the study of Fourier series, particularly in the study of the conjugate series, is too well known to require further elaboration here.

Littlewood and Paley [6] found certain means of derivatives of functions in $H_{p}$ to be of special importance in obtaining estimates of sums of Fourier series. These means, $g(\theta)$ and $g^{*}(\theta)$, are defined by the formulae

$$
g(\theta)=g(\theta, \phi)=\left\{\int_{0}^{1}(1-\rho)\left|\phi^{\prime}\left(\rho e^{i \theta}\right)\right|^{2} d \rho\right\}^{1 / 2},
$$

and

$$
g^{*}(\theta)=g^{*}(\theta, \phi)=\left\{\frac{1}{\pi} \int_{0}^{1}(1-\rho) d \rho \int_{0}^{2 \pi}\left|\phi^{\prime}\left(\rho e^{i t}\right)\right|^{2} P(\rho, t-\theta) d t\right\}^{1 / 2}
$$

where $P(\rho, t)$ denotes the Poisson kernel

$$
\frac{1}{2} \cdot \frac{1-\rho^{2}}{1-2 \rho \cos t+\rho^{2}}
$$

If $\phi \in H_{p}, p>0$, the nontangential limit of $\phi(z)$ is known [9] to exist at almost every point of $|z|=1$, and we have then that

$$
\lim _{\rho \rightarrow 1}\left\{\frac{1}{2 \pi} \int_{0}^{2 \pi}\left|\phi\left(\rho e^{i \theta}\right)\right|^{p} d \theta\right\}^{1 / p}=\left\{\frac{1}{2 \pi} \int_{0}^{2 \pi}\left|\phi\left(e^{i \theta}\right)\right|^{p} d \theta\right\}^{1 / p},
$$

which value we denote by $\|\phi\|_{p}$. The Littlewood-Paley functions have been shown to have the following relation to the original functions,

Presented to the Society, December 29, 1953; received by the editors April 18, 1955. 


$$
\begin{aligned}
& A_{p}\|\phi\|_{p} \leqq\|g\|_{p} \leqq A_{p}\|\phi\|_{p}, \\
& A_{p}\|\phi\|_{p} \leqq\left\|g^{*}\right\|_{p} \leqq A_{p}\|\phi\|_{p} ;
\end{aligned}
$$

the symbol \|\|$_{p}$ denotes the usual norm in $L_{p}$ except when used as indicated above, and the $A_{p}$ 's denote, as they will throughout this paper, various positive constants dependent on the indicated subscripts. The left-hand inequalities require, of course, that

$$
\phi(0)=0 .
$$

The inequality for $g^{*}(\theta)$ was shown by Littlewood and Paley only for $p$ an even integer and was extended to $p>1$ by Zygmund [10]; the right-hand inequality for $g(\theta)$ was demonstrated (although not explicitly stated) by Littlewood and Paley for all $p>0$, that on the left for $p>1$.

In the study of functions analytic in a half-plane it is of interest to define a class analogous to $H_{p}$. Throughout this paper we shall use the same notation for functions in circles and half-planes, distinguishing between them by means of their arguments. If $\phi(s), s=\sigma+i \tau$, now denotes a function analytic in the half-plane $R(s)>0$, it is said to be in the Hille-Tamarkin class $\mathcal{F}_{p}$, $p \geqq 1$, if

$$
\int_{-\infty}^{\infty}|\phi(\sigma+i \tau)|^{p} d \tau
$$

is uniformly bounded in $\sigma[5]$. As in the circle, the nontangential limit exists almost everywhere as $\sigma \rightarrow 0$ and we define

$$
\|\phi\|_{p}=\left\{\int_{-\infty}^{\infty}|\phi(0+i \tau)|^{p} d \tau\right\}^{1 / p}
$$

which is, of course, also

$$
\lim _{\sigma \rightarrow 0}\left\{\left\{\int_{-\infty}^{\infty}|\phi(\sigma+i \tau)| p d \tau\right\}^{1 / p}\right.
$$

The Poisson kernel for the half-plane, $P(\sigma, \tau)$, is merely

$$
\frac{\sigma}{\sigma^{2}+\tau^{2}}
$$

If $f(\tau)$ denotes a function of class $L_{p}, p>1$, in $(-\infty, \infty)$, its conjugate function, $\tilde{f}(\tau)$, is given by

$$
\frac{1}{\pi} \int_{-\infty}^{\infty} \frac{f(x)}{\tau-x} d x
$$

the integral being taken in the principal value sense. The conjugate function is known to be in class $L_{p}[9]$, and 


$$
\|\tilde{f}\|_{p} \leqq A_{p}\|f\|_{p}
$$

The function

$$
\phi(s)=\frac{1}{\pi} \int_{-\infty}^{\infty}[f(x)+i \tilde{f}(x)] P(\sigma, \tau-x) d x
$$

is analytic in the half-plane $\sigma>0$, and as in the case of the unit circle, is of class $\mathfrak{H C}_{p}$. If $\phi(s)$ is given as in $\mathfrak{K}_{p}, p \geqq 1$, then it is the Poisson integral of its boundary values, and even more, it admits of a Cauchy integral representation

$$
\phi(s)=\frac{1}{2 \pi} \int_{-\infty}^{\infty} \frac{\phi(i x)}{s-i x} d x .
$$

If $\|\phi(\sigma+i \tau)\|_{p}$ denotes

$$
\left\{\int_{-\infty}^{\infty}|\phi(\sigma+i \tau)| p d \tau\right\}^{1 / p}
$$

so that

$$
\|\phi\|_{p}=\|\phi(0+i \tau)\|_{p},
$$

we may show that $\|\phi(\sigma+i \tau)\|_{p}$ is a continuous monotone decreasing function of $\sigma$ and that

$$
\lim _{\sigma \rightarrow \infty}\|\phi(\sigma+i \tau)\|_{p}=0 .
$$

Quite elementary arguments show that

$$
|\phi(\sigma+i \tau)| \leqq[\pi \sigma]^{-1 / p}\|\phi\|_{p}
$$

and for $\delta>0$,

$$
\lim _{\rho \rightarrow \infty}\left|\phi\left(\delta+\rho e^{i \theta}\right)\right|=0, \quad-\pi / 2 \leqq \theta \leqq \pi / 2,
$$

uniformly in $\theta$.

A Möbius transformation mapping $\sigma>0$ into the unit circle takes the class $\mathfrak{H}_{p}$ into a subclass of $H_{p}[1]$. Many of the important properties of functions of class $\mathfrak{H}_{p}$ can be deduced from corresponding results for functions of class $H_{p}$ by means of this class inclusion. Other results, as, for example, the validity of the Cauchy integral representation, seem to require a direct proof. The results we consider here are of this type.

We propose here to define analogues of the Littlewood-Paley functions for functions analytic in a half-plane. It will be seen that exactly analogous results may be obtained.

In addition to the Littlewood-Paley functions we shall consider the 
analogue of a function which has been studied by Lusin, Marcinkiewicz, and Zygmund [7]. They consider a function of obvious geometrical significance. Let $\Omega$ be a region in the unit circle bounded by a single closed curve which touches the circle at $z=1$ only, and nontangentially there. Thus all points of $\Omega$ are contained between two chords intersecting at $z=1$ to form an angle $\eta<\pi$. $\Omega_{\theta}$ will denote this region rotated until $z=1$ coincides with $e^{i \theta}$. We define $s(\theta)$ to be the square root of the area of the image of $\Omega_{\theta}$ on the Riemann surface of $\phi$. Thus

$$
s(\theta)=\left\{\iint_{\Omega_{\theta}}\left|\phi^{\prime}(z)\right|^{2} d \omega\right\}^{1 / 2} .
$$

It is known that

$$
A_{p, \eta}\|\phi\|_{p} \leqq\|s\|_{p} \leqq A_{p, \eta}\|\phi\|_{p}
$$

for all $p>0$ on the right, and for all $p>1$ on the left, just as for the function $g(\theta)$. This result, too, may be extended to functions analytic in the half-plane.

A separate section of this paper is devoted to each function, $\$ 2$ fcr $g$, $\S 3$ for $s, \S 4$ for $g^{*}$. It is clear that the three functions are very closely related and each section will use the results of those before it.

2 . Let $\phi(s)$ denote a function analytic in $R(s)>0$. We define

$$
g(\tau)=g(\tau, \phi)=\left\{\int_{0}^{\infty} \sigma\left|\phi^{\prime}(s)\right|^{2} d \sigma\right\}^{1 / 2} .
$$

It will be convenient to adopt the following notation:

$$
\begin{array}{ll}
\phi_{q}(s)=\phi(q+s), & q \geqq 0, \\
g_{q}(\tau)=g\left(\tau, \phi_{q}\right)=\left\{\int_{q}^{\infty}(\sigma-q)\left|\phi^{\prime}(s)\right|^{2} d \sigma\right\}^{1 / 2} . &
\end{array}
$$

Capitalization of the letter denoting a function and change of the argument to $\tau$ will denote the introduction of a function of Hardy-Littlewood maximal type. For example,

$$
\Phi(\tau)=\max _{s \in R_{\tau}}|\phi(s)|
$$

where

$$
R_{\tau}=\left[s \mid s=i \tau+\rho e^{i \theta}, \rho>0,-\alpha<\theta<\alpha<\pi / 2\right] .
$$

This convention will be observed throughout this paper.

We shall enumerate several lemmas to which we shall frequently refer in that which follows.

LemMa 1. If $\phi(s)$ is in class $\mathfrak{H}_{p}, p>1$, then

$$
\|\Phi\|_{p} \leqq A_{p}\|\phi\|_{p}
$$


This is an analogue of a well known maximal theorem of Hardy and Littlewood [3]. Its proof is quite similar to that of the classical result and is due to E. Trombley [8].

LemMa 2. If $\phi(s)$ is in class $\mathfrak{H}_{p}, p \geqq 1$, then

(i) $\phi^{\prime}(s)=\frac{1}{2 \pi} \int_{-\infty}^{\infty} \frac{\phi(\alpha+i \beta)}{(\alpha+i \beta-s)^{2}} d \beta$, $\sigma>\alpha>0$

(ii) $\phi^{\prime}(s)=o(1)$ as $|s| \rightarrow \infty$ uniformly in every half-plane, $\sigma>\alpha>0$,

(iii) $\phi^{\prime}(s)=O\left[(\sigma-\alpha)^{-(1+p) / p}\right]$ as $\sigma \rightarrow \infty$.

Lemma 3. If $\phi(s)$ is in class $\mathfrak{K}_{p}, p \geqq 1$, then

$$
\phi(s)=B(s) W(s)
$$

where $B(s)$ is analytic in $R(s)>0,|B| \leqq 1$, and $\lim _{\sigma \rightarrow 0}|B|=1$ almost everywhere; $W(s)$ is in class $\mathfrak{H}_{p}$ and has no zeros in $R(s)>0$.

LEMMA 4. If $\phi(s)$ is in class $\mathfrak{K}_{p}, p \geqq 1$, then $g_{q}(\tau)$ is bounded for any $q>0$ and

$$
\begin{aligned}
g_{q} & \leqq g \\
\|g\|_{p} & =\lim _{q \rightarrow 0}\left\|g_{q}\right\|_{p} .
\end{aligned}
$$

We shall prove these in sequence.

It is evident that if the Cauchy integral exists then

$$
\frac{1}{2 \pi} \int_{-\infty}^{\infty} \frac{\phi(\alpha+i \beta)}{(\alpha+i \beta-s)^{2}} d \beta
$$

exists as well. Then

$$
\begin{aligned}
\phi^{\prime}(s)= & \lim _{h \rightarrow 0}\left\{\frac{1}{2 \pi} \cdot \frac{1}{h} \int_{-T}^{T} \phi(\alpha+i \beta)\left[\frac{1}{\alpha+i \beta-(s+h)}-\frac{1}{\alpha+i \beta-s}\right] d \beta\right. \\
& +A(h, T)\}
\end{aligned}
$$

where $A(h, T)=o(1)$ as $T \rightarrow \infty$ uniformly in small $h$. Thus

$$
\phi^{\prime}(s)=\frac{1}{2 \pi} \int_{-T}^{T} \frac{\phi(\alpha+i \beta)}{(\alpha+i \beta-s)^{2}} d \beta+o(1)
$$

as $T \rightarrow \infty$, and an application of the Weierstrass double-series theorem gives the desired result.

Applying Hölder's inequality to the Cauchy integral representation of $\phi^{\prime}(s)$, we have 


$$
\begin{aligned}
\left|\phi^{\prime}(s)\right| & \leqq \frac{1}{2 \pi} \int_{-\infty}^{\infty} \frac{|\phi(\alpha+i \beta)|}{(\sigma-\alpha)^{2}+(\beta-\tau)^{2}} d \beta \\
& \leqq A\left\{\int_{-\infty}^{\infty} \frac{|\phi(\alpha+i \beta)|^{p}}{(\sigma-\alpha)^{2}+(\beta-\tau)^{2}} d \beta\right\}^{1 / p}(\sigma-\alpha)^{-(p-1) / p}
\end{aligned}
$$

Choose $T$ such that

$$
\left(\int_{-\infty}^{-T}+\int_{T}^{\infty}\right)|\phi(\alpha+i \beta)|^{p} d \beta<\delta
$$

and note that

$$
\frac{1}{(\sigma-\alpha)^{2}+(\beta-\tau)^{2}}<\frac{1}{\alpha^{2}} \text { for } \sigma>2 \alpha .
$$

Then the contribution of the extreme parts of the integral is less than $\delta / \alpha^{2}$. Clearly

$$
\int_{-T}^{T} \frac{|\phi(\alpha+i \beta)|^{p}}{(\sigma-\alpha)^{2}+(\beta-\tau)^{2}}=O\left(\frac{1}{|s|^{2}}\right)
$$

uniformly in the half-plane $\sigma>2 \alpha$, and consideration of these estimates yields the second part of Lemma 2 for $p>1$; the proof is clearly much simpler for $p=1$ and need not be given here.

Proceeding in a similar manner

$$
\begin{aligned}
\left|\phi^{\prime}(s)\right|(\sigma-\alpha) & \leqq \frac{1}{2 \pi} \int_{-\infty}^{\infty} \frac{|\phi(\alpha+i \beta)|(\sigma-\alpha)}{(\sigma-\alpha)^{2}+(\beta-\tau)^{2}} d \beta \\
& \leqq A(\sigma-\alpha)^{-1 / p}\left\{\int_{-\infty}^{\infty} \frac{|\phi(\alpha+i \beta)| p(\sigma-\alpha)^{2}}{(\sigma-\alpha)^{2}+(\beta-\tau)^{2}} d \beta\right\}^{1 / p} \\
& \leqq A(\sigma-\alpha)^{-1 / p}\|\phi(\alpha+i \beta)\|^{p}
\end{aligned}
$$

or

$$
\left|\phi^{\prime}(s)\right| \leqq A\|\phi\|_{p}(\sigma-\alpha)^{-(1+p) / p}
$$

and since the demonstration is trivialized in case $p=1$, we have completed the proof of Lemma 2 .

Let us now map $\sigma>0$ into $|z|<1$ by the Möbius transformation

$$
s=\frac{1-z}{1+z}
$$

and write

$$
\phi(s)=\zeta(z) .
$$


Then there is a well known theorem of F. Riesz to the effect that

$$
\zeta(z)=\mathscr{B}(z) \mathscr{W}(z)
$$

where $\mathbb{B}$ and $\mathscr{W}$ have properties analogous to those required of $B$ and $W$. The inverse transformation clearly preserves the properties of $B$ and we shall designate $\mathbb{B}(z(s))$ by $B(s)$. That $W(s)$, which corresponds to $\mathscr{W}(z)$, is an element of $\mathcal{K}_{p}$ follows from the fact that the Poisson integral for $W(s)$ along $\sigma=0$ is equal to the Poisson integral for $\mathscr{W}(z)$ around the unit circle for corresponding values of $z$ and $s$. It is not necessary to impose any restriction on the zeros of $\phi(s)$.

We note now that

$$
\begin{aligned}
g_{q}^{2}(\tau) & =\int_{q}^{\infty}(\sigma-q)\left|\phi^{\prime}(s)\right|^{2} d \sigma \\
& \leqq A \int_{q}^{\infty}(\sigma-q)(\sigma-q / 2)^{-2(1+p) / p} d \sigma \\
& \leqq A<\infty
\end{aligned}
$$

by Lemma 2 (iii). Also

$$
\begin{aligned}
g_{q}^{2}(\tau) & =\int_{q}^{\infty}(\sigma-q)\left|\phi^{\prime}(s)\right|^{2} d \sigma \\
& \leqq \int_{q}^{\infty} \sigma\left|\phi^{\prime}(s)\right|^{2} d \sigma \\
& \leqq g^{2}(\tau) .
\end{aligned}
$$

As $q \rightarrow 0, g_{q} \rightarrow g$, and so by Fatou's lemma,

$$
\|g\|_{p}=\lim _{\boldsymbol{Q} \rightarrow 0}\left\|g_{q}\right\|_{p}
$$

Our lemmas now established, we proceed to the main theorem.

TheOREM 1. Let $\phi(s)$ be a function of class $\mathfrak{F}_{p}, p \geqq 1$. If

$$
g(\tau)=g(\tau, \phi)=\left\{\int_{0}^{\infty} \sigma\left|\phi^{\prime}(s)\right|^{2} d \sigma\right\}^{1 / 2}
$$

then $g(\tau)$ is in class $L_{p}$ and

$$
\|g\|_{p} \leqq A_{p}\|\phi\|_{p} .
$$

Conversely, if $\phi(s)$ is analytic in $R(s)>0, \phi(s) \rightarrow 0$ as $s \rightarrow \infty$ uniformly in every half-plane $R(s)>\epsilon>0$, and $g(\tau)$ is in class $L_{p}, p>1$, then $\phi(s)$ is in class $\mathcal{F}_{p}$ and 
174

DANIEL WATERMAN

January

$$
\|\phi\|_{p} \leqq A_{p}\|g\|_{p} .
$$

The first part of this result will be demonstrated for even $p$, giving explicit details for $p=4$ and showing how the proof may be extended to $p=2 k$, $k=1,2, \cdots$. From the even integers it will then be extended to all $p \geqq 1$. In the proof $\phi(s)$ will denote $\phi_{q}(s)$, for it actually suffices to prove the result in this case. For if

$$
\left\|g_{q}\right\|_{p} \leqq A_{p}\left\|\phi_{q}\right\|_{p}
$$

then

$$
\left\|g_{q}\right\|_{p} \leqq A_{p}\left\|\phi_{q}\right\|_{p} \leqq A_{p}\|\phi\|_{p},
$$

letting $q \rightarrow 0$, applying Lemma 4 , and comparing the extreme terms we would have

$$
\|g\|_{p} \leqq A_{p}\|\phi\|_{p}
$$

the desired result.

We shall show now that

$$
\|g\|_{4} \leqq A\|\phi\|_{4}
$$

under the assumption that $\phi$ is in $\mathfrak{F C}_{4}$.

Let us write

$$
\begin{aligned}
\|g\|_{4}^{4} & =\int_{-\infty}^{\infty} g^{4} d \tau \\
& =\int_{-\infty}^{\infty}\left(\int_{0}^{\infty} \sigma\left|\phi^{\prime}(s)\right|^{2} d \sigma\right)^{2} d \tau \\
& =\int_{-\infty}^{\infty} \int_{0}^{\infty} \int_{0}^{\infty} \sigma_{1} \sigma_{2}\left|\phi_{1}^{\prime}\right|^{2}\left|\phi_{2}^{\prime}\right|^{2} d \sigma_{1} d \sigma_{2} d \tau
\end{aligned}
$$

where

$$
\begin{aligned}
& \phi_{1}=\phi\left(\sigma_{1}+i \tau\right), \\
& \phi_{2}=\phi\left(\sigma_{2}+i \tau\right) .
\end{aligned}
$$

Then we have

$$
\|g\|_{4}^{4}=A \int_{-\infty}^{\infty} \iint_{0 \leqq \sigma_{1} \leqq \sigma_{2} \leqq \infty} \sigma_{1} \sigma_{2}\left|\phi_{1}^{\prime}\right|^{2}\left|\phi_{2}^{\prime}\right|^{2} d \sigma_{1} d \sigma_{2} d \tau .
$$

We make the substitution

$$
\left|\phi_{1}^{\prime}\right|^{2}=\Delta\left|\phi_{1}\right|^{2} / 4=\left\{\left|\phi_{1}\right|_{\sigma_{1} \sigma_{1}}^{2}+\left|\phi_{1}\right|_{\tau \tau}^{2}\right\} / 4 .
$$

Then 


$$
\begin{aligned}
& \int_{-M}^{N} \iint_{0 \leqq \sigma_{1} \leqq \sigma_{2} \leqq C} \sigma_{1} \sigma_{2}\left|\phi_{1}^{\prime}\right|^{2}\left|\phi_{2}^{\prime}\right|^{2} d \sigma_{1} d \sigma_{2} d \tau \\
& \quad \leqq \int_{-M}^{N} \iint_{0 \leqq \sigma_{1} \leqq \sigma_{2} \leqq C}\left(\sigma_{1} \sigma_{2}\left|\phi_{2}^{\prime}\right|^{2}\left|\phi_{1}\right|_{\sigma_{1} \sigma_{1}}^{2}+\sigma_{1} \sigma_{2}\left|\phi_{2}^{\prime}\right|^{2}\left|\phi_{1}\right|_{\tau \tau}^{2}\right) d \sigma_{1} d \sigma_{2} d \tau \\
&=P+Q .
\end{aligned}
$$

Partial integration of $P$ with respect to $\sigma_{1}$, yields

$$
P \leqq \int_{-M}^{N} \int_{0}^{C}\left(\sigma_{2}^{2}\left|\phi_{2}\right|_{\sigma_{2}}^{2}\left|\phi_{2}^{\prime}\right|^{2}+\sigma_{2}\left|\phi_{2}^{\prime}\right|^{2}|\phi(0+i \tau)|^{2}\right) d \sigma_{2} d \tau=P_{1}+P_{2}
$$

Clearly

$$
|\phi(0+i \tau)| \leqq \Phi(\tau)
$$

and

$$
|\phi|_{\sigma}^{2}=2|\phi||\phi|_{\sigma} \leqq 2\left|\phi \phi^{\prime}\right|
$$

Writing $\phi^{\prime}$ as a Cauchy integral we have

$$
\left|\phi^{\prime}\right|=\frac{1}{2 \pi}\left|\int_{C} \frac{\phi(z)}{(\sigma+i \tau-z)^{2}} d z\right|
$$

where $C$ is a circle about $\sigma+i \tau$ with radius $\delta=k \sigma$ contained in the angular region given in the definition of $\Phi$. Then

$$
\begin{aligned}
\left|\phi^{\prime}\right| & \leqq \frac{1}{2 \pi} \Phi \int_{C} \frac{1}{\delta^{2}} d z \\
& \leqq A \Phi / \sigma
\end{aligned}
$$

and we note that in a similar fashion we can obtain

$$
\left|\phi^{\prime \prime}\right| \leqq A \Phi / \sigma^{2} \text {. }
$$

Since this result is of considerable use in what follows we state it as a lemma.

LEMMA 5. If $\phi(s)$ is analytic in the half-plane $R(s)>0$, then

$$
\left|\phi^{\prime}\right| \leqq A \Phi / \sigma, \quad\left|\phi^{\prime \prime}\right| \leqq A \Phi / \sigma^{2} .
$$

Applying Lemma 5 to $P_{1}$ we have

$$
\begin{aligned}
P_{1} & \leqq A \int_{-M}^{N} \int_{0}^{C}\left(\sigma_{2}^{2}\left|\phi_{2}\right|\left|\phi_{2}^{\prime}\right|^{2} \Phi / \sigma_{2}\right) d \sigma_{2} d \tau \\
& \leqq A \int_{-M}^{N} \Phi^{2}\left(\int_{0}^{C} \sigma\left|\phi^{\prime}\right|^{2} d \sigma\right) d \tau
\end{aligned}
$$

Since 


$$
P_{2}=\int_{-M}^{N}|\phi(0+i \tau)|^{2}\left(\int_{0}^{c} \sigma\left|\phi^{\prime}\right|^{2} d \sigma\right) d \tau
$$

we have

$$
P \leqq A \int_{-M}^{N} \Phi^{2}\left(\int_{0}^{C} \sigma\left|\phi^{\prime}\right|^{2} d \sigma\right) d \tau
$$

If in $Q$ we invert the order of integration and integrate by parts with respect to $\tau$ we havel

$$
\begin{aligned}
Q= & \iint_{0 \leqq \sigma_{1} \leqq \sigma_{2} \leqq C} \int_{-M}^{N}\left|\phi_{2}^{\prime}\right|^{2}\left|\phi_{1}\right|_{\tau \tau}^{2} d \tau d \sigma_{1} d \sigma_{2} \\
= & \iint_{0 \leqq \sigma_{1} \leqq \sigma_{2} \leqq C} \sigma_{1} \sigma_{2}\left[\left|\phi_{2}^{\prime}\right|^{2}\left|\phi_{1}\right|_{\tau}^{2}\right]_{-M}^{N} d \sigma_{1} d \sigma_{2} \\
& -\int_{-M}^{N} \iint_{0 \leqq \sigma_{1} \leqq \sigma_{2} \leqq C} \sigma_{1} \sigma_{2}\left|\phi_{2}^{\prime}\right|_{\tau}^{2}\left|\phi_{1}\right|_{\tau}^{2} d \sigma_{1} d \sigma_{2} d \tau=Q_{1}+Q_{2} .
\end{aligned}
$$

Applying Lemma 5,

$$
\begin{aligned}
\left|Q_{2}\right| & \leqq A \int_{-M}^{N} \iint_{0 \leqq \sigma_{1} \leqq \sigma_{2} \leqq C} \sigma_{1} \sigma_{2}\left|\phi_{2}^{\prime}\right|\left|\phi_{2}^{\prime \prime}\right|\left|\phi_{1}\right|\left|\phi_{1}^{\prime}\right| d \sigma_{1} d \sigma_{2} d \tau \\
& \leqq A \int_{-M}^{N} \iint_{0 \leqq \sigma_{1} \leqq \sigma_{2} \leqq C}\left(\sigma_{1} \sigma_{2}\left|\phi_{1}^{\prime}\right|\left|\phi_{2}^{\prime}\right| \Phi^{2} / \sigma_{2}^{2}\right) d \sigma_{1} d \sigma_{2} d \tau \\
& \leqq A \int_{-M}^{N} \Phi^{2} \iint_{0 \leqq \sigma_{1} \leqq \sigma_{2} \leqq c} \frac{\sigma_{1}}{\sigma_{2}}\left|\phi_{1}^{\prime}\right|\left|\phi_{2}^{\prime}\right| d \sigma_{1} d \sigma_{2} d \tau \\
& \leqq A \int_{-M}^{N} \Phi^{2}\left\{\int_{0}^{C} \int_{\sigma_{1}}^{C}\left|\phi_{1}^{\prime}\right|^{2} \frac{\sigma_{1}^{2}}{\sigma_{2}^{2}} d \sigma_{1} d \sigma_{2}+\int_{0}^{C}\left|\phi_{2}^{\prime}\right|^{2}\left(\int_{0}^{\sigma_{2}} d \sigma_{1}\right) d \sigma_{2}\right\} d \tau \\
& \leqq A \int_{-M}^{N} \Phi^{2}\left(\int_{0}^{C} \sigma\left|\phi^{\prime}\right|^{2} d \sigma\right) d \tau .
\end{aligned}
$$

Consider now

$$
\begin{aligned}
&\left|\iint_{0 \leqq \sigma_{1} \leqq \sigma_{2} \leqq C} \sigma_{1} \sigma_{2}\left[\left|\phi_{2}^{\prime}\right|^{2}\left|\phi_{1}\right|_{\tau}^{2}\right]_{|\tau|=B} d \sigma_{1} d \sigma_{2}\right| \\
& \leqq 2 \iint_{0 \leqq \sigma_{1} \leqq \sigma_{2} \leqq C} \sigma_{1} \sigma_{1}\left[\left|\phi_{2}^{\prime}\right|^{2}\left|\phi_{1}\right|\left|\phi_{1}^{\prime}\right|\right]_{|\tau|=B} d \sigma_{1} d \sigma_{2}=o(1)
\end{aligned}
$$

as $B \rightarrow \infty$ for $C$ fixed, in the light of the estimates of $|\phi|$ and $\left|\phi^{\prime}\right|$ in the lemmas. Combining the results on $Q_{1}$ and $Q_{2}$ with those obtained for $P_{1}$ and $P_{2}$ we have 


$$
\int_{-M}^{N} \iint_{0 \leqq \sigma_{1} \leqq \sigma_{2} \leqq C} \sigma_{1} \sigma_{2}\left|\phi_{1}^{\prime}\right|^{2}\left|\phi_{2}^{\prime}\right|^{2} d \sigma_{1} d \sigma_{2} d \tau
$$

$$
\leqq A \int_{-M}^{N} \Phi^{2}\left(\int_{0}^{C} \sigma\left|\phi^{\prime}\right|^{2} d \sigma\right) d \tau+o(1)
$$

as $M, N \rightarrow \infty$ for fixed $C$. Let

$$
\int_{0}^{c} \sigma\left|\phi^{\prime}\right|^{2} d \sigma=c g^{2}(\tau)
$$

Then

$$
\int_{-M}^{N} c g^{4} d \tau \leqq A \int_{-M}^{N} \Phi^{2} \cdot{ }_{c} g^{2} d \tau+o(1) .
$$

Applying Hölder's inequality and dividing by $\left\{\int_{-M}^{N} \operatorname{co} g^{4} d \tau\right\}^{1 / 2}$,

$$
\left\{\int_{-M}^{N} c g^{4} d \tau\right\}^{1 / 2} \leqq A\left\{\int_{-M}^{N} \Phi^{4} d \tau\right\}^{1 / 2}+o(1) .
$$

Letting $M, N \rightarrow \infty$ and applying Lemma 1, we have

$$
\left\{\int_{-\infty}^{\infty} c g^{4} d \tau\right\}^{1 / 2} \leqq A\left\{\int_{-\infty}^{\infty}|\phi|^{4} d \tau\right\}^{1 / 2}
$$

Letting $C \rightarrow \infty$ and taking the square root of both sides yields

$$
\|g\|_{4} \leqq A\|\phi\|_{4}
$$

the desired result.

In the case $p=2 k$ we consider

$$
\int_{-M}^{N} \int_{0 \leqq \sigma_{1} \leqq \sigma_{2} \leqq \ldots \leqq \sigma_{k} \leqq C}^{\cdots} \int \prod_{1}^{k} \sigma_{n}\left|\phi_{n}^{\prime}\right|^{2} \prod_{1}^{k} d \sigma_{n} d \tau .
$$

We make the Laplacian substitution and evaluate the first integral

$$
\int_{-M}^{N} \int_{0 \leqq \sigma_{1} \leqq \cdots \leqq C}^{\ldots} \int \prod_{1}^{k} \sigma_{n} \prod_{2}^{k}\left|\phi_{n}^{\prime}\right|^{2} \cdot\left|\phi_{1}\right|_{\sigma_{1} \sigma_{1}}^{2} \prod_{1}^{k} d \sigma_{n} d \tau
$$

in precisely the same manner as in the case $k=2$ to yield

$$
\int_{-M}^{N} \Phi^{2} \int_{0 \leqq \sigma_{2} \leqq \cdots \leqq \sigma_{k} \leqq C} \underset{2}{\cdots} \prod_{2}^{k} \sigma_{n}\left|\phi_{n}^{\prime}\right|^{2} \prod_{2}^{k} d \sigma_{n} d \tau .
$$

The second part

$$
\int_{-M}^{N} \int_{0 \leqq \sigma_{1} \leqq \cdots \leqq}^{\cdots} \int \cdots\left|\phi_{1}\right|_{\tau \tau}^{2} \prod_{1}^{k} d \sigma_{n} d \tau
$$


is integrated by parts as in the case $k=2$. The part

$$
\int_{0 \leqq \sigma_{1} \leqq \ldots \leqq c} \ldots \int \prod_{1}^{k} \sigma_{n}\left[\left|\phi_{1}\right|_{r}^{2} \prod_{2}^{k}\left|\phi_{n}^{\prime}\right|^{2}\right]_{-M}^{N} \prod_{1}^{k} d \sigma_{n}=o(1)
$$

for fixed $C$ as $M, N \rightarrow \infty$. The part

$$
\begin{aligned}
\int_{-M}^{N} \int \cdots & \int \prod_{1}^{k} \sigma_{n}\left(\prod_{2}^{k}\left|\phi_{n}^{\prime}\right|^{2}\right)_{\tau}\left|\phi_{1}^{2}\right|_{r} \prod_{1}^{k} d \sigma_{n} d \tau \\
& =\int_{-M}^{N} \int \cdots \int \prod_{1}^{k} \sigma_{n}\left|\phi_{1}^{2}\right| \tau\left\{\sum_{i=2}^{k}\left|\phi_{i}^{\prime}\right|_{\tau}^{2} \prod_{2, n \neq i}^{k}\left|\phi_{n}^{\prime}\right|^{2}\right\} \prod_{1}^{k} d \sigma_{n} d \tau
\end{aligned}
$$

which is less in absolute value than

$$
\begin{aligned}
A \int_{-M}^{N} \int \cdots \int \prod_{1}^{k} \sigma_{n}\left|\phi_{1}\right|\left|\phi_{1}^{\prime}\right|\left\{\sum_{i=2}^{k}\left|\phi_{i}^{\prime}\right|\left|\phi_{i}^{\prime \prime}\right| \prod_{2, n \neq i}^{k}\left|\phi_{n}^{\prime}\right|^{2}\right\} \prod_{1}^{k} d \sigma_{n} d \tau \\
\leqq A \int_{-M}^{N} \Phi^{2} \int \cdots \int \prod_{1}^{k} \sigma_{n}\left|\phi_{1}^{\prime}\right|\left\{\sum_{i=2}^{k} \frac{1}{\sigma_{i}^{2}}\left|\phi_{i}^{\prime}\right| \prod_{2, n \neq i}^{k}\left|\phi_{n}^{\prime}\right|^{2}\right\} \prod_{1}^{k} d \sigma_{n} d \tau
\end{aligned}
$$

by Lemma 5 . Consider one term in this sum

$$
\begin{aligned}
\int_{-M}^{N} \Phi^{2} \int \cdots & \int \prod_{1}^{k} \sigma_{n}\left|\phi_{1}^{\prime}\right| \frac{1}{\sigma_{i}^{2}}\left|\phi_{i}^{\prime}\right| \prod_{2, n \neq i}^{k}\left|\phi_{n}^{\prime}\right|^{2} \prod_{1}^{k} d \sigma_{n} d \tau \\
& =\int_{-M}^{N} \Phi^{2} \int \cdots \int\left(\left(\prod_{1, n \neq i}^{k} \sigma_{n}\right) / \sigma_{i}\right)\left|\dot{\phi}_{1}\right|\left|\phi_{i}^{\prime}\right| \prod_{2, n \neq i}^{k}\left|\phi_{n}^{\prime}\right|^{2} \prod_{1}^{k} d \sigma_{n} d \tau \\
& \leqq \int_{-M}^{N} \Phi^{2}\left(\iint_{0 \leqq \sigma_{1} \leqq \sigma_{i} \leqq c} \frac{\sigma_{1}}{\sigma_{2}}\left|\phi_{1}^{\prime}\right|\left|\phi_{i}^{\prime}\right| d \sigma_{1} d \sigma_{i}\right) \\
& \cdot\left(\int_{0 \leqq \sigma_{2} \leqq \ldots \leqq \sigma_{i-1} \leqq \sigma_{i}{ }_{1} \leqq \ldots \leqq c} \int \prod_{2, n \neq i}^{k} \sigma_{n}\left|\phi_{n}^{\prime}\right|^{2} \prod_{2, n \neq i}^{k} d \sigma_{n}\right) d \tau \\
\leqq & A_{k} \int_{-M}^{N} \Phi^{2}\left(\int_{0}^{c} \sigma\left|\phi^{\prime}\right|{ }^{2} d \sigma\right)^{k-1} d \tau .
\end{aligned}
$$

Hence

$$
\begin{aligned}
\int_{-M}^{N} c g^{2 k} d \tau & \leqq A_{k} \int_{-M}^{N} \Phi^{2} c g^{2 k-2} d \tau+o(1) \\
& \leqq A_{k}\left\{\int_{-M}^{N} \Phi^{2 k} d \tau\right\}^{1 / k}\left\{\int_{-M}^{N} c g^{2 k} d \tau\right\}^{(k-1) / k}+o(1) .
\end{aligned}
$$

Dividing by the factor on the right containing $\mathrm{g}$, letting $M, N$ and then $C$ approach infinity, taking the square root and applying Lemma 1, we have 


$$
\|g\|_{2 k} \leqq A_{k}\|\Phi\|_{2 k} \leqq A_{k}\|\phi\|_{2 k}
$$

which was to be shown.

We must now show that

$$
\|g\|_{p} \leqq A_{p}\|\phi\|_{p}
$$

for all $p \geqq 1$.

By Lemma 3 we may write

$$
\phi=\phi_{1}+\phi_{2}, \quad \phi_{1}=(B-1) W, \quad \phi_{2}=W .
$$

Thus $\phi_{1}$ and $\phi_{2}$ have no zeros in $R(s)>0$, are in $\mathfrak{C}_{p}$, and satisfy

Since

$$
\begin{aligned}
& \left\|\phi_{1}\right\|_{p} \leqq 2\|\phi\|_{p}, \\
& \left\|\phi_{2}\right\|_{p}=\|\phi\|_{p} .
\end{aligned}
$$

$$
g\left(\phi_{1}+\phi_{2}\right) \leqq A\left\{g\left(\phi_{1}\right)+g\left(\phi_{2}\right)\right\}
$$

we need only prove the result in the case where $\phi$ has no zeros.

Let

$$
\phi=\omega^{y}
$$

where $y=2 k / p$ and $2 k$ is the least even integer such that $2 k \geqq 2 p$.

Then $\omega(s)$ is a regular function and is in $\mathcal{F C}_{2 k}$. We have

$$
\begin{aligned}
\phi^{\prime} & =y \omega^{y-1} \omega^{\prime}, \\
\left|\phi^{\prime}\right|^{2} & \leqq A|\omega|^{2(y-1)}\left|\omega^{\prime}\right|^{2},
\end{aligned}
$$

and

$$
\begin{aligned}
g^{2}(\tau, \phi) & \leqq A \sup _{\sigma>0}|\omega|^{2(y-1)} \cdot \int_{0}^{\infty} \sigma\left|\omega^{\prime}\right|^{2} d \sigma \\
& =A \Omega^{2(\gamma-1)} g^{2}(\tau, \omega) .
\end{aligned}
$$

Thus

$$
g^{p}(\tau, \phi) \leqq A_{p} \Omega^{p(y-1)} g^{p}(\tau, \omega)
$$

But

$$
p y=2 k \text { and } p(y-1)=2 k / y^{\prime}
$$

Thus

$$
\int_{-\infty}^{\infty} g^{p}(\tau, \phi) d \tau \leqq A_{p} \int_{-\infty}^{\infty} \Omega^{2 k / y^{\prime}} g^{p}(\tau, \omega) d \tau
$$

Applying our theorem for even exponents to the right integral 


$$
\begin{aligned}
\int_{-\infty}^{\infty} \Omega^{2 k / y^{\prime}} g^{p}(\tau, \omega) d \tau & \leqq\left\{\int_{-\infty}^{\infty} \Omega^{2 k} d \tau\right\}^{1 / y^{\prime}}\left\{\int_{-\infty}^{\infty} g^{p \nu}(\tau, \omega) d \tau\right\}^{1 / \nu} \\
& \leqq A_{p}\left\{\int_{-\infty}^{\infty} \Omega^{2 k} d \tau\right\}^{1 / y^{\prime}}\left\{\int_{-\infty}^{\infty}|\omega|^{2 k} d \tau\right\}^{1 / \nu} \\
& \leqq A_{p}\left\{\int_{-\infty}^{\infty}|\omega|^{2 k} d \tau\right\}^{1 / y^{\prime}}\left\{\int_{-\infty}^{\infty}|\omega|^{2 k} d \tau\right\}^{1 / \nu} \\
& =A_{p} \int_{-\infty}^{\infty}|\omega|^{2 k} d \tau .
\end{aligned}
$$

Thus

$$
\int_{-\infty}^{\infty} g^{p}(\tau, \phi) d \tau \leqq A_{p} \int_{-\infty}^{\infty}|\omega|^{2 k} d \tau=A_{p} \int_{-\infty}^{\infty}|\phi|^{p} d \tau
$$

Taking the $p$ th root of the extreme terms we have established

$$
\|g\|_{p} \leqq A_{p}\|\phi\|_{p}
$$

the first part of Theorem 1.

The second part of Theorem 1 is proven by conjugacy with the opposite inequality. Our $\phi(s)$ is now analytic in $R(s)>0$ and has the property that its limit is zero as $s$ approaches infinity in $R(s)>\epsilon>0$. We shall assume that a right translation of axes to $(\eta, 0)$ has been made and so our function is analytic in the half-plane $R(s)>-\eta$. The function $g(\tau)$ exists, and by hypothesis and Lemma 1

$$
\|g\|_{p}<\infty
$$

We define

$$
\begin{aligned}
& f(\tau)=\mathbb{R}[\phi(0+i \tau)], \\
& h(\tau)=h_{N}(\tau)=|f|^{p-1}(\text { signum } f) \chi_{N}(\tau),
\end{aligned}
$$

where $\chi_{N}$ is continuous and

$$
\chi_{N}(\tau)=\left\{\begin{array}{rlrl}
1, & & |\tau| & \leqq N-1, \\
0, & |\tau| & \geqq N, \\
<1, & N-1 & <|\tau|<N .
\end{array}\right.
$$

Further we let

$\psi=$ the $\phi$ associated with $h$, i.e., the analytic function whose real part is the Poisson integral of $h$,

$$
\gamma=g(\tau, \psi), \quad u=R(\phi), \quad u^{*}=R(\psi) .
$$

We consider the equality 


$$
\left.\left.\int_{\epsilon}^{K} \sigma\left(u u^{*}\right)_{\sigma \sigma} d \sigma=\sigma\left(u u^{*}\right)_{\sigma}\right]_{\epsilon}^{K}-\left(u u^{*}\right)\right]_{\epsilon}^{K},
$$

obtained by an integration by parts and note that:

$$
\left|K u_{\sigma}(K, \tau) u^{*}(K, \tau)\right|=o(1) \frac{K}{K^{2}+\tau^{2}}
$$

as $K \rightarrow \infty$, for by evaluating $\phi^{\prime}$ as a Cauchy integral around a circle of radius $C K(0<C<1)$ and center $K+i \tau$ and writing $u^{*}$ as the Poisson integral of $h(\tau)$ we have at once that our expression is

$$
\leqq K \sup _{\mid \varepsilon-(K+\{\tau) \mid=C K}|\phi(s)| \cdot \frac{1}{C K} \cdot \frac{N A K}{K^{2}+\min _{0 \leqq \beta \leqq N}(|\tau|-\beta)^{2}}
$$

and so

$$
=o(1) \frac{K}{K^{2}+\tau^{2}}
$$

for $K$ sufficiently large.

$$
\begin{aligned}
\left|K u_{\sigma}^{*}(K, \tau) u(K, \tau)\right| & =o(1) \frac{K}{K^{2}+\tau^{2}}, \\
\left|u(K, \tau) u^{*}(K, \tau)\right| & =o(1) \frac{K}{K^{2}+\tau^{2}},
\end{aligned}
$$

by arguments similar to that employed in (i).

$$
\begin{aligned}
\left|\epsilon u_{\sigma}(\epsilon, \tau) u^{*}(\epsilon, \tau)\right| & \leqq \epsilon A \sup _{C}|\phi| \sup _{(s)>0}\left|u^{*}\right| \\
& =o(1) \text { uniformly in } \tau \text { as } \epsilon \rightarrow 0,
\end{aligned}
$$

the $C$ designating a circle of radius $\eta / 2$ about $(\epsilon+i \tau)$.

(v)

$$
\begin{aligned}
\left|\epsilon u_{\sigma}^{*}(\epsilon, \tau) u(\epsilon, \tau)\right| & \leqq\left|\epsilon u_{\sigma}^{*}(\epsilon, \tau)\right| \sup _{R(s)>0}|\phi| \\
& =o(1) \text { uniformly in } \tau \text { as } \epsilon \rightarrow 0 .
\end{aligned}
$$

We shall show that $\left|\sigma u_{\sigma}^{*}(\sigma, \tau)\right| \rightarrow 0$ uniformly in $\tau$ as $\sigma \rightarrow 0$.

From the Poisson integral representation we have

$$
\begin{aligned}
\left|u_{\sigma}^{*}(\sigma, \tau)\right| & =\frac{1}{\pi}\left|\int_{-\infty}^{\infty} h(x)\left(\frac{\sigma}{\sigma^{2}+(x-\tau)^{2}}\right) d x\right| \\
& =\frac{1}{\pi}\left|\int_{-\infty}^{\infty} h(x+\tau) \frac{x^{2}-\sigma^{2}}{\left(\sigma^{2}+x^{2}\right)^{2}} d x\right| .
\end{aligned}
$$


Choose $\delta>0$ such that

$$
\left|h(\tau)-h\left(\tau^{\prime}\right)\right|<\epsilon \text { if }\left|\tau-\tau^{\prime}\right|<\delta .
$$

Note that $|h(\tau)|<A<\infty$. Then for $\sigma<\delta$

$$
\begin{aligned}
\left|\left(\int_{\delta}^{\infty}+\int_{-\infty}^{-\delta}\right) h(x+\tau) \frac{x^{2}-\sigma^{2}}{\left(\sigma^{2}+x^{2}\right)^{2}} d x\right| & <A \int_{\delta}^{\infty} \frac{x^{2}-\sigma^{2}}{\left(\sigma^{2}+x^{2}\right)^{2}} d x \\
<A / \delta &
\end{aligned}
$$

Write

$$
\int_{-\delta}^{\delta} h(x+\tau) \frac{\sigma^{2}-x^{2}}{\left(\sigma^{2}+x^{2}\right)^{2}} d x=\int_{-\delta}^{\delta}(h(\tau)+k(x)) \frac{\sigma^{2}-x^{2}}{\left(\sigma^{2}+x^{2}\right)^{2}} d x .
$$

Then

$$
\int_{-\delta}^{\delta} h(\tau) \frac{\sigma^{2}-x^{2}}{\left(\sigma^{2}+x^{2}\right)^{2}} d x \leqq A / \delta
$$

and if we divide the other integral into several parts

$$
\int_{-\delta}^{\delta} k(x) \frac{\sigma^{2}-x^{2}}{\left(\sigma^{2}+x^{2}\right)^{2}} d x=\left(\int_{-\delta}^{\sigma}+\int_{\sigma}^{\delta}\right)+\int_{-\sigma}^{\sigma}=P+Q
$$

we have

$$
\begin{aligned}
|P| & \leqq \\
& \left.<\int_{-\delta}^{-\sigma}+\int_{+o}^{\delta}\right)|k(x)| \frac{x^{2}-\sigma^{2}}{\left(\sigma^{2}+x^{2}\right)^{2}} d x \\
|Q| & <A \epsilon / \sigma .
\end{aligned}
$$

Thus

$$
\left|u_{\sigma}^{*}(\sigma, \tau)\right| \leqq A\left(\frac{1}{\delta}+\frac{\epsilon}{\sigma}\right)
$$

and so

$$
\left|\sigma u_{\sigma}^{*}(\sigma, \tau)\right| \leqq A\left(\frac{\sigma}{\delta}+\epsilon\right)
$$

for small $\sigma$, which proves (v).

Substituting these estimates in our integration by parts we have

$$
\int_{0}^{K} \sigma\left(u u^{*}\right)_{\sigma \sigma} d \sigma=u(0, \tau) u^{*}(0, \tau)+o(1) \frac{K}{K^{2}+\tau^{2}}
$$

for $K$ large. 
We have, therefore, for any $\mathcal{N}>N$,

$$
\begin{aligned}
\int_{-\infty}^{\infty} f h d \tau & =\int_{-N}^{N} f h d \tau \\
& =\int_{-\mathcal{N}}^{\mathcal{N}} f h d \tau \\
& =\int_{-}^{\mathcal{N}} \int_{0}^{K} \sigma\left(u u^{*}\right)_{\sigma \sigma} d \sigma d \tau+\int_{-\mathcal{N}}^{\mathcal{N}} o(1) \frac{K}{K^{2}+\tau^{2}} d \tau \\
& \leqq \int_{0}^{K} \sigma\left|\int_{-}^{\mathcal{N}}\left(u u^{*}\right)_{\sigma \sigma} d \tau\right| d \sigma+o(1)
\end{aligned}
$$

as $K \rightarrow \infty$.

Consider

$$
\int_{-\mathcal{N}}^{\mathcal{N}}\left(u u^{*}\right)_{\sigma \sigma} d \tau=\int_{-\mathcal{N}}^{\mathcal{N}}\left(u_{\sigma \sigma} u^{*}+2 u_{\sigma} u_{\sigma}^{*}+u u_{\sigma \sigma}^{*}\right) d \tau
$$

We shall estimate the first and last parts. We have

$$
\left.\int_{-}^{\mathcal{N}} u^{\mathcal{N} \sigma} u^{*} d \tau=-\int_{-\mathcal{N}}^{\mathcal{N}} u_{\tau \tau} u^{*} d \tau=-u_{\tau} u^{*}\right]_{-}^{\mathcal{N}}+\int_{-}^{\mathcal{N}} u^{\mathcal{N}} u_{\tau}^{*} d \tau
$$

and similarly

$$
\left.\int_{-\mathcal{N}}^{\mathcal{N}} u_{\sigma \sigma}^{*} u d \tau=-u_{\tau}^{*} u\right]_{-\mathcal{N}}^{\mathcal{N}}+\int_{-\mathcal{N}}^{\mathcal{N}} u_{r} u_{\tau}^{*} d \tau
$$

We note next that $\sigma\left|u_{\tau} u^{*}\right|$ and $\sigma\left|u_{\tau}^{*} u\right|$ are $o(1)$ as $|\tau| \rightarrow \infty$ uniformly in $\sigma$. Hence

$$
\begin{aligned}
\int_{-\infty}^{\infty} f h d \tau \leqq & 2 \int_{0}^{K} \sigma \int_{-\mathcal{N}}^{\mathcal{N}}\left(\left|u_{\tau} u_{\tau}^{*}\right|+\left|u_{\sigma} u_{\sigma}^{*}\right|\right) d \tau d \sigma \\
& \left.\left.+\int_{0}^{K} \sigma\left(\mid u_{\tau}^{*} u\right]_{-}^{\mathcal{N}}|+| u_{\tau} u^{*}\right]_{-\mathcal{N}}^{\mathcal{N}} \mid\right) d \sigma+o(1) .
\end{aligned}
$$

Allowing $\mathcal{N}$ and then $K$ to approach infinity, we have

$$
\begin{aligned}
\int_{-\infty}^{\infty} f h d \tau & \leqq 2 \int_{0}^{\infty} \sigma \int_{-\infty}^{\infty}\left(\left|u_{\tau} u_{\tau}^{*}\right|+\left|u_{\sigma} u_{\sigma}^{*}\right|\right) d \tau d \sigma \\
& \leqq 4 \int_{0}^{\infty} \sigma \int_{-\infty}^{\infty}\left|\phi^{\prime} \psi^{\prime}\right| d \tau d \sigma .
\end{aligned}
$$

\section{Thus}




$$
\begin{aligned}
\int_{-\infty}^{\infty} f h d \tau & \leqq A \int_{-\infty}^{\infty}\left[\int_{0}^{\infty} \sigma\left|\phi^{\prime}\right|^{2} d \sigma\right]^{1 / 2}\left[\int_{0}^{\infty} \sigma\left|\psi^{\prime}\right|^{2} d \sigma\right]^{1 / 2} d \tau \\
& =A \int_{-\infty}^{\infty} g(\tau) \gamma(\tau) d \tau \\
& \leqq A\|g\|_{p}\|\gamma\|_{p^{\prime}} \\
& \leqq A_{p}\|g\|_{p}\|\psi\|_{p^{\prime}} \\
& \leqq A_{p}\|g\|_{p}\left\{\int_{-\infty}^{\infty}|f|^{p} \chi_{N}^{p^{\prime}} d \tau\right\}^{1 / p^{\prime}}
\end{aligned}
$$

by an application of the first part of Theorem 1 and the fact that

$$
\|\psi\|_{p^{\prime}} \leqq A_{p^{\prime}}\|R(\psi)\|_{p^{\prime}}
$$

Then

$$
\int_{-\infty}^{\infty}|f|^{0} \chi_{N} d \tau \leqq A_{p}\|g\|_{p}\left\{\int_{-\infty}^{\infty}|f|^{z} \chi_{N} d \tau\right\}^{1 / p^{\prime}}
$$

or

$$
\left\{\int_{-\infty}^{\infty}|f|^{p} \chi_{N} d \tau\right\}^{1 / p} \leqq A_{p}\|g\|_{p}
$$

Letting $N \rightarrow \infty$ we have

$$
\|f\|_{p} \leqq A_{p}\|g\|_{p}
$$

and analogously

$$
\|\tilde{f}\|_{p} \leqq A_{p}\|g\|_{p}
$$

Thus

$$
\|\phi\|_{p} \leqq A_{p}\|g\|_{p}
$$

and consideration of Lemma 1 yields the same result for the original function.

3. We turn now to the analogue of $s(\theta)$. Let $\Omega$ denote the region in the half-plane $R(s)>0$ between the lines $\tau= \pm k \sigma$. The vertical translate of this angular region will be denoted by $\Omega_{\tau}$ where $0+i \tau$ is the new vertex. Then if $\phi(s)$ is analytic in $R(s)>0$ we define

$$
s(\tau)=\left\{\iint_{\Omega_{\tau}}\left|\phi^{\prime}(s)\right|^{2} d \omega\right\}^{1 / 2}
$$

We shall establish the following theorem.

TheORem 2. Let $\phi(s)$ be a function of class $\mathfrak{H}_{p}, p \geqq 1$. If 


$$
s(\tau)=\left\{\iint_{\Omega_{\tau}}\left|\phi^{\prime}(s)\right|^{2} d \omega\right\}^{1 / 2}
$$

then $s(\tau)$ is in class $L_{p}$ and

$$
\|s\|_{p} \leqq A_{p, k}\|\phi\|_{p}
$$

Conversely, if $\phi(s)$ is analytic in $R(s)>0, \phi(s) \rightarrow 0$ as $s \rightarrow \infty$ uniformly in every half-plane $R(s)>\epsilon>0$, and $s(\tau)$ is in class $L_{p}, p>1$, then $\phi(s)$ is in class $\mathcal{F}_{p}$ and

$$
\|\phi\|_{p} \leqq A_{p, k}\|s\|_{p \cdot}
$$

We note that to demonstrate the latter part of this theorem we need only show that

$$
g(\tau) \leqq A_{k} s(\tau),
$$

for then an application of Theorem 1 would yield the result. This is easily accomplished. We shall demonstrate it for $\tau=0$ to simplify notation, but this restriction will be seen to be superfluous.

Consider the intervals

$$
\left[\frac{1}{2^{n}}, \frac{1}{2^{n+1}}\right], \quad n=0,1,2, \cdots
$$

We denote by $\sigma_{n}$ the point in the $n$th interval where $\left|\phi^{\prime}\right|$ attains its maximum. Hence

$$
\begin{aligned}
\int_{0}^{1} \sigma\left|\phi^{\prime}\right|^{2} d \sigma & \leqq \sum_{n=0}^{\infty}\left|\phi^{\prime}\left(\sigma_{n}\right)\right|^{2} \int_{1 / 2^{n+1}}^{1 / 2^{n}} \sigma d \sigma \\
& =\frac{3}{8} \sum_{0}^{\infty} \frac{\left|\phi^{\prime}\left(\sigma_{n}\right)\right|^{2}}{2^{2 n}} .
\end{aligned}
$$

We note that

$$
\left|\phi^{\prime}\left(\sigma_{n}\right)\right|^{2} \leqq \frac{1}{2 \pi} \int_{0}^{2 \pi}\left|\phi^{\prime}\left(\sigma_{n}+\rho e^{i \theta}\right)\right|^{2} d \theta
$$

and so if $R_{n}$ designates the radius of a circle $C\left(\sigma_{n}, R_{n}\right)$ about $\sigma_{n}$,

$$
\frac{1}{2} R_{n}^{2}\left|\phi^{\prime}\left(\sigma_{n}\right)\right|^{2} \leqq \frac{1}{2 \pi} \iint_{C\left(\sigma_{n}, R_{n}\right)}\left|\phi^{\prime}\right|^{2} d \omega .
$$

Let $R_{n}=\delta 2^{-n}$, where $\delta$ is chosen depending on $k$ alone, so that $C\left(\sigma_{n}, R_{n}\right)$ is in $\Omega$. We note that no area can ever be jointly contained in more than two circles. Then 


$$
\frac{\left|\phi^{\prime}\left(\sigma_{n}\right)\right|^{2}}{2^{2 n}} \leqq \frac{1}{\pi \delta^{2}} \iint_{C\left(\sigma_{n}, R_{n}\right)}\left|\phi^{\prime}\right|^{2} d \omega
$$

or

$$
\begin{aligned}
\int_{0}^{1} \sigma\left|\phi^{\prime}\right|^{2} d \sigma & \leqq \frac{3}{8 \pi \delta^{2}} \sum_{n=0}^{\infty} \iint_{C\left(\sigma_{n}, R_{n}\right)}\left|\phi^{\prime}\right|^{2} d \omega \\
& \leqq \frac{3}{4 \pi \delta^{2}} \iint_{\Omega}\left|\phi^{\prime}\right|^{2} d \omega .
\end{aligned}
$$

If we now consider the intervals $\left[2^{n}, 2^{n+1}\right], n=0,1, \cdots$, and define $\sigma_{n}$ to be the point in the $n$th interval where $\left|\phi^{\prime}\right|$ attains a maximum, then

$$
\int_{1}^{\infty} \sigma\left|\phi^{\prime}\right|^{2} d \delta \leqq \sum_{0}^{\infty} \mid \phi^{\prime}\left(\left.\sigma_{n}^{\prime}\right|^{2} \int_{2^{n}}^{2 n+1} \sigma d \sigma=\frac{3}{2} \sum_{0}^{\infty}\left|\phi^{\prime}\left(\sigma_{n}\right)\right|^{2} 2^{2 n} .\right.
$$

Let $R_{n}=\delta 2^{n}$ where $\delta$ is chosen so that $C\left(\sigma_{n}, R_{n}\right)$ is within $\Omega$. Then, as above,

$$
\begin{aligned}
\int_{1}^{\infty} \sigma\left|\phi^{\prime}\right|^{2} d \sigma & \leqq \frac{3}{2 \pi \delta^{2}} \sum_{0}^{\infty} \iint_{C\left(\sigma_{n}, R_{n}\right)}\left|\phi^{\prime}\right|^{2} d \omega \\
& \leqq \frac{3}{\pi \delta^{2}} \iint_{\Omega}\left|\phi^{\prime}\right|^{2} d \omega .
\end{aligned}
$$

Combining this with the previous result we have

$$
\int_{0}^{\infty} \sigma\left|\phi^{\prime}\right|^{2} d \sigma \leqq A_{k} \iint_{\Omega}\left|\phi^{\prime}\right|^{2} d \omega
$$

which establishes the latter half of our theorem.

In the proof of the first part of the theorem we shall require the following lemma, an extension of a result of Hardy and Littlewood [2] due to E. Trombley [8].

Lemma 6. If $f(\tau) \in L_{p}, p>1$, in $(-\infty, \infty)$ and

$$
f^{*}(\tau)=\sup _{A>0} \frac{1}{2 A} \int_{\tau-A}^{\tau+A} f(x) d x
$$

then $\left\|f^{*}\right\|_{p} \leqq A_{p}\|f\|_{p}$.

For a complex function $\phi(\sigma+i \tau), \phi^{*}(\tau)$ shall denote $|\phi(0+i \tau)|^{*}$.

Let us write $S(\tau)=s^{2}(\tau)$. Then

$$
\|s\|_{p}=\left\|S^{1 / 2}\right\|_{p}=\max _{h \in X_{\mu}}\left\{\int_{-\infty}^{\infty} S(\tau) h(\tau) d \tau\right\}^{1 / 2}
$$

where 


$$
\mu=\left(\frac{1}{2} p\right)^{\prime}, \quad X_{\mu}=\left\{h(\tau)>0 \ni \int_{-\infty}^{\infty}|h| \mu d \tau \leqq 1\right\}
$$

Consider a fixed $h(\tau)$. Then if $\bar{\tau}=k \sigma$,

$$
\begin{aligned}
\int_{-\infty}^{\infty} S(\tau) h(\tau) d \tau & =\int_{-\infty}^{\infty} h\left(\tau^{\prime}\right)\left\{\iint_{\Omega_{\tau^{\prime}}}\left|\phi^{\prime}\right|{ }^{2} d \sigma d \tau\right\} d \tau^{\prime} \\
& =\int_{-\infty}^{\infty} h\left(\tau^{\prime}\right)\left\{\int_{0}^{\infty} d \sigma \int_{\tau^{\prime}-\bar{\tau}}^{\tau^{\prime} \bar{\tau}}\left|\phi^{\prime}\right|^{2} d \tau\right\} d \tau^{\prime} \\
& =\int_{0}^{\infty} d \sigma \int_{-\infty}^{\infty} 2 \bar{\tau}\left|\phi^{\prime}\right|^{2} d \tau\left\{\frac{1}{2 \bar{\tau}} \int_{-\infty}^{\infty} h\left(\tau^{\prime}\right) \chi\left(\tau-\tau^{\prime}, \bar{\tau}\right) d \tau^{\prime}\right\} .
\end{aligned}
$$

$\chi(a, b)$ denotes here the characteristic function of the interval $[-b, b]$ with variable $a$. Then

$$
\begin{aligned}
\int_{-\infty}^{\infty} S(\tau) h(\tau) d \tau & \leqq A_{k} \int_{0}^{\infty} \sigma d \sigma \int_{-\infty}^{\infty} \mid \phi^{\prime}{ }^{2} h^{*}(\tau) d \tau \\
& =A_{k} \int_{-\infty}^{\infty} h^{*}(\tau)\left(\int_{-\infty}^{\infty} \sigma\left|\phi^{\prime}\right|{ }^{2} d \sigma\right) d \tau \\
& =A_{k} \int_{-\infty}^{\infty} h^{*}(\tau) g^{2}(\tau) d \tau .
\end{aligned}
$$

Applying Hölder's inequality to the right-hand side

$$
\begin{aligned}
\int_{-\infty}^{\infty} S(\tau) h(\tau) d(\tau) & \leqq A_{k}\left\|h^{*}\right\|_{\mu}\|g\|_{p}^{2} \\
& \leqq A_{k}\|g\|_{p}^{2} .
\end{aligned}
$$

Thus for $p \geqq 2$ we may apply Theorem 1 and we have

$$
\|s\|_{p} \leqq A_{k}\|g\|_{p} \leqq A_{p, k}\|\phi\|_{p} .
$$

We must now extend the result to $p \geqq 1$.

Since a decomposition of the function $\phi$ into two nonzero summands may be effected precisely as in the proof of Theorem 1 with precisely analogous implications we may assume at the onset that $\phi(s)$ has no zeros. We write then

$$
\psi^{4 / p}(s)=\phi(s)
$$

clearly $\psi$ is in $F_{4}$ and

$$
\|s(\tau, \psi)\|_{4} \leqq A_{k}\|\psi\|_{4}
$$

Forming $s(\tau, \phi)$ we have 


$$
\phi^{\prime}(s)=4 / p[\psi(s)]^{4 / p-1} \cdot \psi^{\prime}(s)
$$

and

$$
s(\tau, \phi) \leqq 4 / p[\Psi(\tau)]^{4 / p-1} s(\tau, \psi)
$$

Then

$$
\begin{aligned}
\int_{-\infty}^{\infty} s^{p}(\tau, \phi) d \tau & \leqq(4 / p)^{p} \int_{-\infty}^{\infty} s^{p}(\tau, \psi) \Psi^{4-p}(\tau) d \tau \\
& \leqq A_{p}\|s(\tau, \psi)\|_{4}^{p}\|\Psi\|_{4}^{4-p} .
\end{aligned}
$$

Applying Lemma 1 and our theorem for $p=4$ we have

$$
\|s(\tau, \phi)\|_{p} \leqq A_{p, k}\|\psi\|_{4}^{4 / p}=A_{p, k}\|\phi\|_{p} .
$$

Thus our theorem is established for all $p \geqq 1$.

4. Our result on $g^{*}(\tau)$ may be stated as follows.

TheOREM 3. Let $\phi$ be a function of class $\mathfrak{H}_{p}, p>1$. If

$$
g^{*}(\tau)=g^{*}(\tau, \phi)=\left\{\frac{1}{\pi} \int_{0}^{\infty} \sigma d \sigma \int_{-\infty}^{\infty}\left|\phi^{\prime}(\sigma+i \beta)\right|{ }^{2} P(\sigma, \tau-\beta) d \beta\right\}^{1 / 2},
$$

then $g^{*}$ is in class $L_{p}$ and

$$
\left\|g^{*}\right\|_{p} \leqq A_{p}\|\phi\|_{p} .
$$

Conversely, if $\phi(s)$ is analytic in $R(s)>0, \phi(s) \rightarrow 0$ uniformly in every half-plane $R(s)>\epsilon>0$ and $g^{*}(\tau)$ is in class $L_{p}, p>1$, then $\phi(s)$ is in class $\mathfrak{H}_{p}$ and

$$
\|\phi\|_{p} \leqq A_{p}\left\|g^{*}\right\|_{p}
$$

Let us suppose that $\Omega_{\tau}$ is a region of the type considered in Theorem 2 . We shall denote its complement with respect to $R(s)>0$ by $\bar{\Omega}_{\tau}$. Then

$$
\begin{aligned}
g^{*^{2}}(\tau)= & \frac{1}{\pi} \iint_{\Omega_{\tau}} \sigma\left|\phi^{\prime}(\sigma+i \beta)\right|^{2} P(\sigma, \tau-\beta) d \beta \\
& +\frac{1}{\pi} \iint_{\bar{\Omega}_{\tau}} \sigma\left|\phi^{\prime}(\sigma+i \beta)\right|{ }^{2} P(\sigma, \tau-\beta) d \beta \\
= & g_{1}^{*^{2}}(\tau)+g_{2}^{*^{2}}(\tau) .
\end{aligned}
$$

In the region $\Omega_{\tau}$

$$
\frac{\sigma^{2}}{\sigma^{2}+(\tau-\beta)^{2}} \leqq 1
$$

and so 


$$
\begin{aligned}
g_{1}^{*^{2}}(\tau) & \leqq \frac{1}{\pi} \iint_{\Omega_{\tau}}\left|\phi^{\prime}(\sigma+i \beta)\right|^{2} d \sigma d \beta \\
& =\frac{1}{\pi} s^{2}(\tau)
\end{aligned}
$$

Hence

$$
g^{*}(\tau) \leqq A\left[s(\tau)+g_{2}^{*}(\tau)\right]
$$

It is also quite clear that in $\Omega_{\tau}$

$$
\frac{\sigma^{2}}{\sigma^{2}+(\tau-\beta)^{2}} \geqq A_{k}
$$

and so

$$
g^{*}(\tau) \geqq A_{k} s(\tau) .
$$

Thus the second part of Theorem 3 follows immediately from the second part of Theorem $2, k$, of course, being taken as constant. We introduce

$$
\begin{aligned}
G^{2}(\tau) & =\int_{0}^{\infty} \int_{|\beta|>k \sigma}\left|\phi^{\prime}(\sigma+i(\beta+\tau))\right|^{2} \frac{\sigma^{2}}{\beta^{2}} d \beta d \sigma \\
& \geqq \frac{1}{\pi} \int_{0}^{\infty} \int_{|\beta|>k \sigma}\left|\phi^{\prime}(\sigma+i(\beta+\tau))\right|^{2} \frac{\sigma^{2}}{\sigma^{2}+\beta^{2}} d \beta d \sigma \\
& =g_{2}^{*^{2}}(\tau) .
\end{aligned}
$$

Let us assume $p \geqq 2$ and write

$$
\|G\|_{p}=\max _{h \in X^{\mu}}\left\{\int_{-\infty}^{\infty} G^{2}(\tau) h(\tau) d \tau\right\}^{1 / 2} .
$$

$X_{\mu}$ is defined as in Theorem 2. Suppose now that $h(\tau)$ is fixed. Let

$$
\chi_{\sigma}(\beta)= \begin{cases}1, & |\beta|>k \sigma \\ 0, & \text { otherwise. }\end{cases}
$$

Then

$$
\begin{aligned}
\int_{-\infty}^{\infty} G^{2}(\tau) h(\tau) d \tau & =\int_{-\infty}^{\infty} h(\tau) d \tau \int_{0}^{\infty} \sigma^{2} d \sigma \int_{-\infty}^{\infty} \frac{\chi_{\sigma}(\beta)}{\beta^{2}}\left|\phi^{\prime}(\sigma+i(\beta+\tau))\right|^{2} d \beta \\
& =\int_{0}^{\infty} \sigma^{2} d \sigma \int_{-\infty}^{\infty}\left|\phi^{\prime}(\sigma+i \beta)\right|^{2} d \beta \int_{-\infty}^{\infty} \frac{h(\tau) \chi_{\sigma}(\beta-\tau)}{(\beta-\tau)^{2}} d \tau
\end{aligned}
$$

We observe that 


$$
\begin{aligned}
\int_{-\infty}^{\infty} \frac{h(\tau) \chi_{\sigma}(\beta-\tau)}{(\beta-\tau)^{2}} d \tau & =\int_{-\infty}^{\infty} \frac{h(\beta-u) \chi_{\sigma}(u)}{u^{2}} d u \\
& =\left(\int_{k \sigma}^{\infty}+\int_{-\infty}^{-k \sigma}\right) \frac{h(\beta-u)}{u^{2}} d u \\
& =\int_{k \sigma}^{\infty}\left([h(\beta+u)+h(\beta-u)] / u^{2}\right) d u
\end{aligned}
$$

Let

$$
H(x, \beta)=\int_{0}^{x}[h(\beta+u)+h(\beta-u)] d u .
$$

Then

$$
H(x, \beta) / x \leqq 2 h^{*}(\beta)
$$

and therefore

$$
H(x, \beta)=o\left(x^{2}\right)
$$

for almost all $\beta$. Integrating by parts

$$
\begin{aligned}
\int_{-\infty}^{\infty} \frac{h(\tau) \chi_{\sigma}(\beta-\tau)}{(\beta-\tau)^{2}} d \tau & \left.=H(u, \beta) / u^{2}\right]_{k \sigma}^{\infty}+A \int_{k \sigma}^{\infty} \frac{H(u, \beta)}{u^{3}} d u \\
& <A \int_{k \sigma}^{\infty} \frac{H(u, \beta)}{u^{3}} d u \\
& \leqq A h^{*}(\beta) \int_{k \sigma}^{\infty} \frac{1}{u^{2}} d u \\
& \leqq A h^{*}(\beta) / \sigma .
\end{aligned}
$$

Thus

$$
\begin{aligned}
\int_{-\infty}^{\infty} G^{2}(\tau) h(\tau) d \tau & \leqq A \int_{0}^{\infty} \sigma d \sigma \int_{-\infty}^{\infty}\left|\phi^{\prime}(\sigma+i \beta)\right|^{2} h^{*}(\beta) d \beta \\
& =A \int_{-\infty}^{\infty} h^{*}(\beta) g^{2}(\beta) d \beta \\
& \leqq A\left\|h^{*}\right\|_{\mu}\left\|_{g}\right\|_{p}^{2} \\
& \leqq A p\|\phi\|_{p}
\end{aligned}
$$

by the application of Lemma 6 and Theorem 1. The extension of this result to $2 \geqq p>1$ is more difficult than the arguments used in the corresponding portion of Theorem 2. We utilize the foHowing lemma.

Lemma 7. If $\phi(s)$ is in class $\mathfrak{K}_{p}, p>1$, and 


$$
\phi_{p}^{*}(\tau)=\{|\phi(0+i \tau)| p\} *^{1 / p}
$$

then

$$
|\phi(\sigma+i(\beta+\tau))| \leqq A_{p} \phi_{p}^{*}(\tau)\left[1+\frac{|\beta|}{\sigma}\right]^{1 / p} .
$$

The proof of this lemma is modeled closely on that of a similar theorem for the unit circle which is due to Hardy and Littlewood [4]. We need only show that

$$
|\phi(\sigma+i(\beta+\tau))| \leqq A \phi^{*}\left[1+\frac{|\beta|}{\sigma}\right],
$$

where $\phi(s)$ could actually be restricted to denote merely the Poisson integral of a function in $L_{1}$ (specifically $\left.|\phi(0 \neq i \tau)|^{p}\right)$, and where by $\phi^{*}$ we mean of course $\phi_{1}^{*}$, for then

$$
\begin{aligned}
|\phi(\sigma+i \beta)|^{p} & \leqq\left\{\frac{1}{\pi} \int_{-\infty}^{\infty} \frac{|\phi(i(\beta+\tau))| \sigma}{\sigma^{2}+\tau^{2}} d \tau\right\}^{p} \\
& \leqq \frac{1}{\pi} \int_{-\infty}^{\infty} \frac{|\phi(i(\beta+\tau))|{ }^{p} \sigma}{\sigma^{2}+\tau^{2}} d \tau
\end{aligned}
$$

by an application of Jensen's theorem. Applying the above

$$
|\phi(\sigma+i(\beta+\tau))|^{p} \leqq A \phi_{p}^{* p}[1+|\beta| / \sigma]
$$

from which the lemma would follow immediately. We introduce the following notation

$$
\begin{aligned}
\phi^{*}(\tau) & =\max _{\Lambda>0}\left\{\frac{1}{2 A} \int_{0}^{\Lambda}(|\phi(i(\tau+\beta))|+|\phi(i(\tau-\beta))|) d \beta\right\} \\
& =\max _{\Lambda>0} \frac{1}{2 A} \widehat{\phi}_{\tau}(A) .
\end{aligned}
$$

Then

$$
\begin{aligned}
\phi(\sigma+i(\beta+\tau)) & =\frac{1}{\pi} \int_{-\infty}^{\infty} \frac{\phi(i u) \sigma}{\sigma^{2}+(u-(\beta+\tau))^{2}} d u \\
& =\frac{1}{\pi} \int_{-\infty}^{\infty} \frac{\phi(i(\tau+u)) \sigma}{\sigma^{2}+(u-\beta)^{2}} d u \\
& =\frac{1}{\pi} \int_{0}^{\infty}\left\{\frac{\phi(i(\tau+u)) \sigma}{\sigma^{2}+(\beta-u)^{2}}+\frac{\phi(i(\tau-u)) \sigma}{\sigma^{2}+(\beta+u)^{2}}\right\} d u
\end{aligned}
$$

Thus 


$$
\begin{aligned}
|\phi(\sigma+i(\beta+\tau))| \leqq & \frac{1}{\pi} \int_{0}^{\infty} \frac{\{|\phi(i(\tau+u))|+|\phi(i(\tau-u))|\} \sigma}{\sigma^{2}+(|\beta|-u)^{2}} d u \\
\leqq & A\left[\widehat{\phi}_{r}(u) \frac{\sigma}{\sigma^{2}+(|\beta|-u)^{2}}\right]_{0}^{\infty} \\
& +A \int_{0}^{\infty} \frac{\widehat{\phi}_{\tau}(u) \sigma|| \beta|-u|}{\left[\sigma^{2}+(|\beta|-u)^{2}\right]^{2}} d u \\
= & A \int_{0}^{\infty} \frac{\widehat{\phi}_{\tau}(u)}{2 u} \frac{u \sigma|| \beta|-u|}{\left[\sigma^{2}+(|\beta|-u)^{2}\right]^{2}} d u .
\end{aligned}
$$

From the definition of $\phi^{*}$ we have then

$$
\begin{aligned}
|\phi(\sigma+i(\beta+\tau))| \leqq & A \phi^{*}(\tau) \int_{0}^{\infty} \frac{u \sigma|| \beta|-u|}{\left[\sigma^{2}+(|\beta|-u)^{2}\right]^{2}} d u \\
\leqq & A \phi^{*}(\tau)\left\{\int_{-\infty}^{\infty} \frac{(|\beta|-u)^{2} \sigma}{\left[\sigma^{2}+(|\beta|-u)^{2}\right]^{2}} d u\right. \\
& \left.+\int_{-\infty}^{\infty} \frac{|\beta| \sigma|| \beta|-u|}{\left[\sigma^{2}+(|\beta|-u)^{2}\right]^{2}} d u\right\} \\
= & A \phi^{*}(\tau)\left\{I_{1}+I_{2}\right\} .
\end{aligned}
$$

But

$$
\begin{aligned}
I_{1} & \leqq \int_{-\infty}^{\infty} \frac{\sigma}{\sigma^{2}+(|\beta|-u)^{2}} d u \\
& =A
\end{aligned}
$$

and

$$
\begin{aligned}
I_{2} & =|\beta| \int_{-\infty}^{\infty} \frac{\sigma|| \beta|-u|}{\left[\sigma^{2}+(|\beta|-u)^{2}\right]^{2}} d u \\
& \leqq|\beta| \int_{-\infty}^{\infty} \frac{d u}{\sigma^{2}+(|\beta|-u)^{2}} \\
& =A \frac{|\beta|}{\sigma} .
\end{aligned}
$$

Substituting these evaluations of $I_{1}$ and $I_{2}$ in the above computation the desired inequality and hence the lemma are immediate.

As in the corresponding portions of the other demonstrations we may assume $\phi$ to have no zeros. Then for $2>p>1$ we write

$$
\phi=\chi^{2 / p}
$$


and the function $\chi(s)$ so defined is in class $\mathfrak{H}_{2}$. Then

$$
\begin{aligned}
\phi^{\prime} & =\frac{2}{p} \chi^{2 / p-1} \chi^{\prime}, \\
\left|\phi^{\prime}\right|^{2} & \leq A|\chi|^{2(2 / p-1)}\left|\chi^{\prime}\right|^{2} .
\end{aligned}
$$

We have then by an application of Lemma 7,

$$
\begin{aligned}
G^{2}(\tau, \phi) & \leqq A \int_{0}^{\infty} \int_{|\beta|>k \sigma}|\chi(\sigma+i(\beta+\tau))|^{2(2 / p-1)}\left|\chi^{\prime}(\sigma+i(\beta+\tau))\right|^{2} \frac{\sigma^{2}}{\beta^{2}} d \beta d \sigma \\
& \left.\leqq A_{p} \chi_{2 / p}^{*^{2(2 / p-1)}} \int_{0}^{\infty} \int_{|\beta|>k \sigma} \mid \beta / \sigma\right)^{2-p}\left(\left.\chi^{\prime}(\sigma+i(\beta+\tau))\right|^{2} \frac{\sigma^{2}}{\beta^{2}} d \beta d \sigma\right. \\
& \leqq A_{p} \chi_{2 / p}^{*^{2(2 / p-1)}} \int_{0}^{\infty} \sigma^{p} \int_{|\beta|>k \sigma}\left(\left|\chi^{\prime}(\sigma+i(\beta+\tau))\right|^{2} /|\beta|^{p}\right) d \beta d \sigma .
\end{aligned}
$$

Raising both sides to the power $p / 2$ and integrating with respect to $\tau$,

$$
\begin{aligned}
\int_{-\infty}^{\infty} G^{p}(\tau) d \tau & \leqq A_{p} \int_{-\infty}^{\infty} \chi_{2 / p}^{*^{2-p}}\left\{\int_{0}^{\infty} \sigma^{p} \int_{|\beta|>k \sigma}\left(\left|\chi^{\prime}(\sigma+i(\beta+\tau))\right|^{2} /|\beta| p\right) d \beta d \sigma\right\}^{p / 2} d \tau \\
\leqq & A_{p}\left\{\int_{-\infty}^{\infty} \chi_{2 / p}^{*^{2}} d \tau\right\}^{2-p / p} \\
& \left\{\int_{-\infty}^{\infty} \int_{0}^{\infty} \int_{|\beta|>k \sigma}\left(\sigma^{p}\left|\chi^{\prime}(\sigma+i(\beta+\tau))\right|^{2} /|\beta|^{p}\right) d \beta d \sigma d \tau\right\}^{p / 2}
\end{aligned}
$$

by the Hölder inequality. Clearly

for

$$
\left\|\chi_{2 / p}^{*}\right\|_{2} \leqq A_{p}\|\chi\|_{2}
$$

$$
\begin{aligned}
\int_{-\infty}^{\infty} \chi_{2 / p}^{*^{2}} d \tau & =\int_{-\infty}^{\infty}\left\{\chi^{2 / p}\right\}^{*^{p}} d \tau \\
& \leqq A_{p} \int_{-\infty}^{\infty}|\chi|^{(2 / p) p} d \tau \\
& =A_{p} \int_{-\infty}^{\infty} \mid \chi\left(0+\left.i \tau\right|^{2} d \tau\right.
\end{aligned}
$$

by Lemma 6 . Also

$$
\int_{-\infty}^{\infty} d \tau \int_{0}^{\infty} \sigma^{p} d \sigma \int_{|\beta|>k \sigma}\left(\left|\chi^{\prime}(\sigma+i(\beta+\tau))\right|^{2} /|\beta| p\right) d \beta \leqq A^{\prime} \chi
$$


for the left-hand expression is but

$$
\begin{aligned}
\int_{-\infty}^{\infty} \int_{0}^{\infty} \int_{|\beta-\tau|>k \sigma}\left(\sigma^{p} \mid\right. & \left.\left.\chi^{\prime}(\sigma+i \tau)\right|^{2} /|\beta-\tau|^{p}\right) d \beta d \sigma d \tau \\
& =\int_{-\infty}^{\infty} \int_{0}^{\infty} \sigma^{p}\left|\chi^{\prime}(\sigma+i \tau)\right|^{2} \int_{|\beta-\tau|>k \sigma}\left(1 /|\beta-\tau|^{p}\right) d \beta d \sigma d \tau \\
& <A \int_{-\infty}^{\infty} \int_{0}^{\infty} \sigma\left|\chi^{\prime}(\sigma+i \tau)\right|^{2} d \sigma d \tau \\
& =A \int_{-\infty}^{\infty} g^{2}(\tau, \chi) d \tau \\
& \leqq A\left\|_{\chi}\right\|_{2}^{2}
\end{aligned}
$$

by Theorem 1 .

Substituting these results in our inequality for $\int_{-\infty}^{\infty} G^{p} d \tau$ we have

$$
\int_{-\infty}^{\infty} G^{p}(\tau, \phi) d \tau \leqq A_{p}\|\chi\|_{2}^{2-p}\|\chi\|_{2}^{p}=A_{p}\|\chi\|_{2}^{2}=A_{p}\|\phi\|_{p}^{p}
$$

for $p>1$.

The required inequality having been obtained for $G(\tau)$ we have

$$
\left\|g^{*}\right\|_{p} \leqq A_{p}\left\{\|s\|_{p}+\|G\|_{p}\right\} \leqq A_{p}\|\phi\|_{p}
$$

for all $p>1$, which is the first part of Theorem 3 .

\section{REFERENCES}

1. R. M. Gabriel, An improved result concerning the zeros of a function regular in a half-plane, J. London Math. Soc. vol. 4 (1929) pp. 307-309.

2. G. H. Hardy and J. E. Littlewood, Some new properties of Fourier constants, Math. Ann. vol. 97 (1926) pp. 159-209.

3. $\longrightarrow$, A maximal theorem with function-theoretic applications, Acta Math. vol. 54 (1930) pp. 81-116. 189.

4. - The strong summability of Fourier series, Fund. Math. vol. 25 (1935) pp. 162-

5. E. Hille and J. D. Tamarkin, On the absolute integrability of Fourier transforms, Fund. Math. vol. 25 (1935) pp. 329-352.

6. J. E. Littlewood and R. E. A. C. Paley, Theorems on Fourier series and power series II. Proc. London Math. Soc. vol. 42 (1937) pp. 52-89.

7. J. Marcinkiewicz and A. Zygmund, On a theorem of Lusin, Duke Math. J. vol. 4 (1938) pp. $473-485$.

8. E. Trombley, Unpublished manuscript.

9. A. Zygmund, Trigonometrical series, Warsaw, 1935.

10. $\longrightarrow$, On certain integrals, Trans. Amer. Math. Soc. vol. 55 (1944) pp. 170-204.

Purdue University, LAFAYETTE, IND. 